\title{
Spin polarization induced facile dioxygen activation in boron-doped graphitic carbon nitride
}

Xinrui $\mathrm{Cao}^{* 1,2}$, Jiacai Shen ${ }^{1}, \mathrm{Xiao}-\mathrm{Fei} \mathrm{Li}^{* 3}$, and $\mathrm{Yi} \mathrm{Luo}^{4}$

${ }^{1}$ Collaborative Innovation Center for Optoelectronic Semiconductors and Efficient Devices, Department of Physics, Xiamen University, Xiamen 361005, China

${ }^{2}$ Fujian Provincial Key Laboratory of Theoretical and Computational Chemistry, Xiamen University, Xiamen 361005, China

${ }^{3}$ School of Optoelectronic Science and Engineering, University of Electronic Science and Technology of China, Chengdu, Sichuan 610054, China

${ }^{4}$ Department of Theoretical Chemistry and Biology, School of Biotechnology, Royal Institute of Technology, S-106 91 Stockholm, Sweden

E-mail: xinruicao@xmu.edu.cn; xf.li@uestc.edu.cn

Table S-1. Predicted lattice parameters, band gap $\left(\mathrm{E}_{\mathrm{g}}\right)$ and magnetic moment $\left(\mu_{\mathrm{B}}\right)$ of B doped gh- $\mathrm{C}_{3} \mathrm{~N}_{4}$.

\begin{tabular}{|c|c|c|c|c|c|}
\hline System & $a(\AA)$ & $b(\AA)$ & $\gamma(\mathrm{deg})$ & $\mathrm{E}_{\mathrm{g}}(\mathrm{eV})$ & $\mathrm{m}\left(\mu_{\mathrm{B}}\right)$ \\
\hline $1 \times 1$ & 7.04 & 7.07 & 120.1 & 0.46 & 1.00 \\
\hline $2 \times 2$ & 13.52 & 13.50 & 118.1 & 0.22 & 1.00 \\
\hline $3 \times 3$ & 20.48 & 20.45 & 120.1 & 0.26 & 3.01 \\
\hline
\end{tabular}


(a)

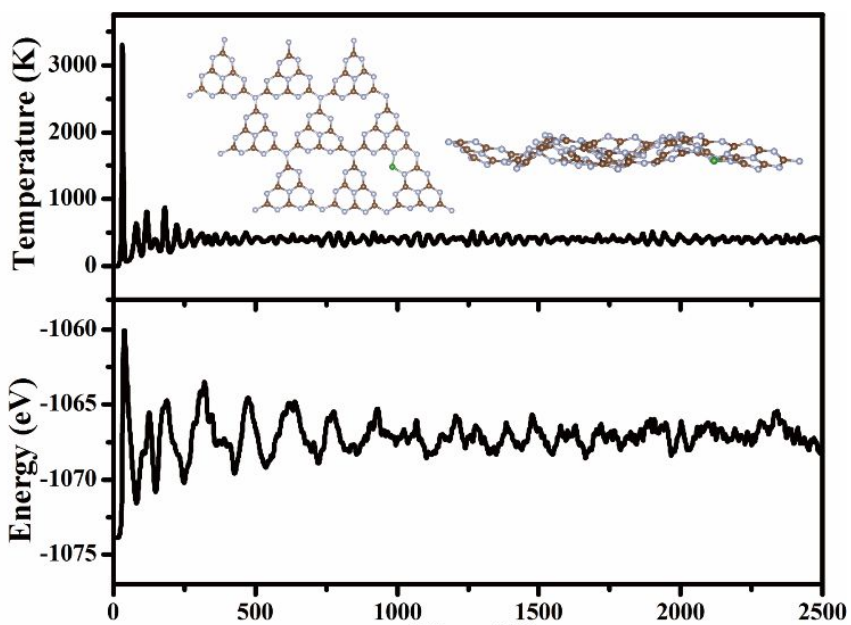

(b)
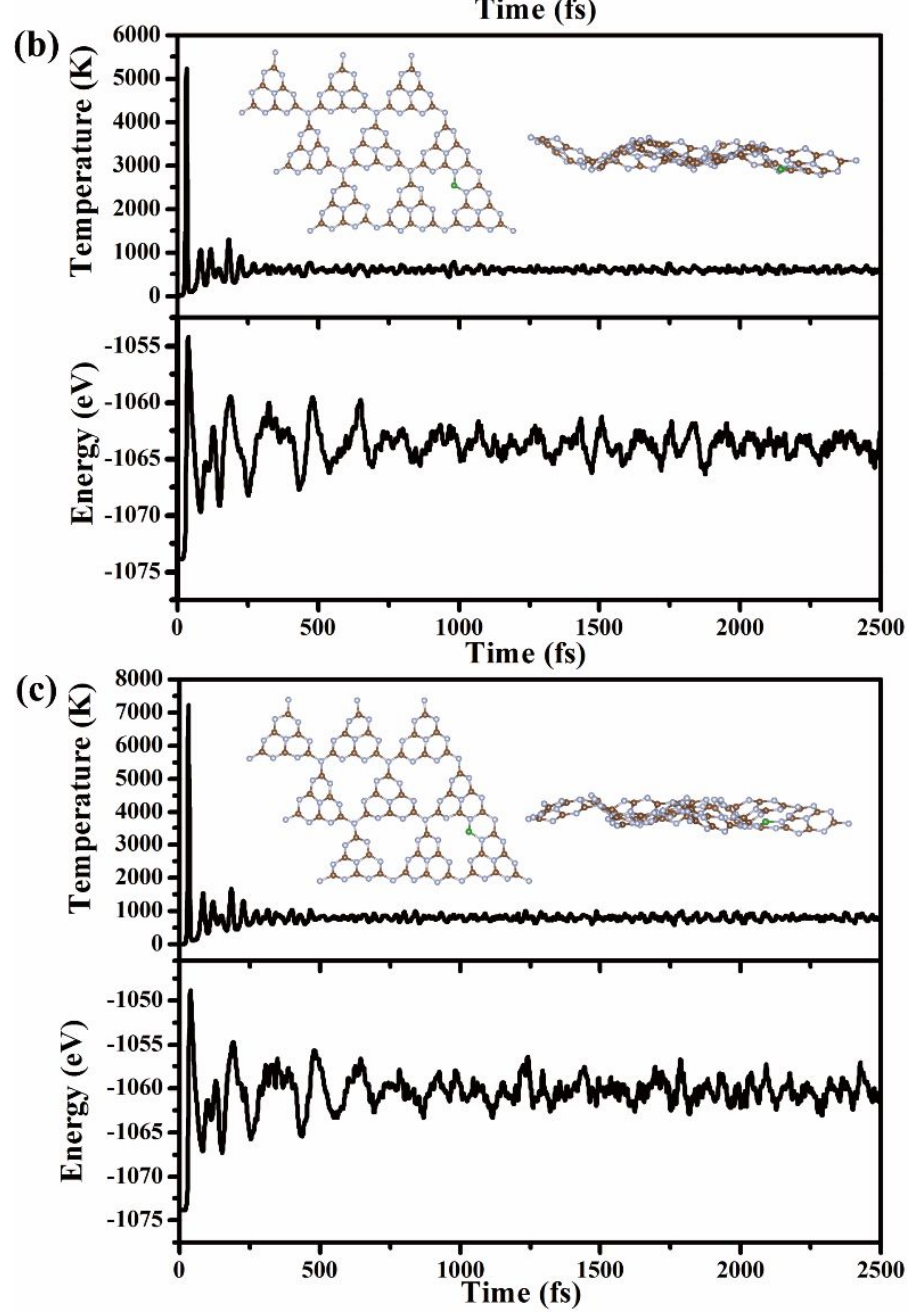

Figure S1. Changes of temperature and energy against the time for AIMD simulations of B/gh- $\mathrm{C}_{3} \mathrm{~N}_{4}$ modeled by a supercell of $(3 \times 3)$ run under different temperature: a) $400 \mathrm{~K}$, b) $600 \mathrm{~K}$ and c) $800 \mathrm{~K}$, inserts are top and side views of the snapshot of atomic configuration at $2.5 \mathrm{ps}$. 
(a)

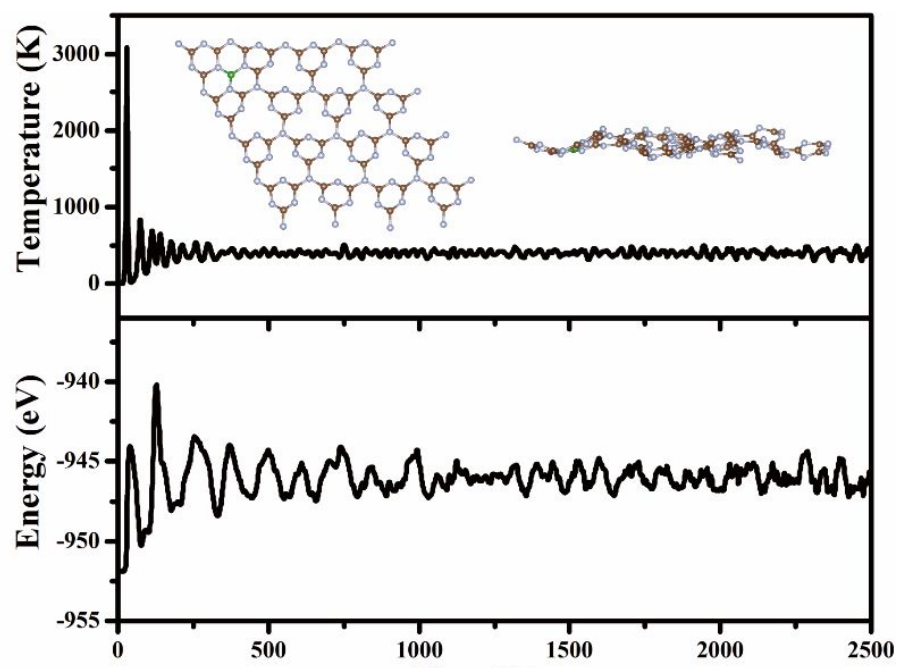

(b)

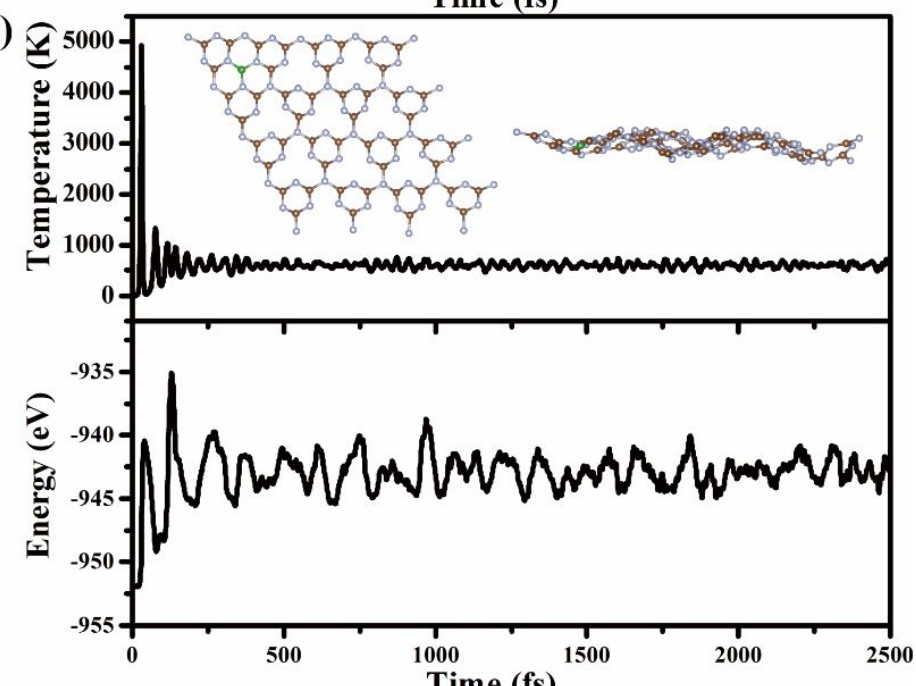

(c)

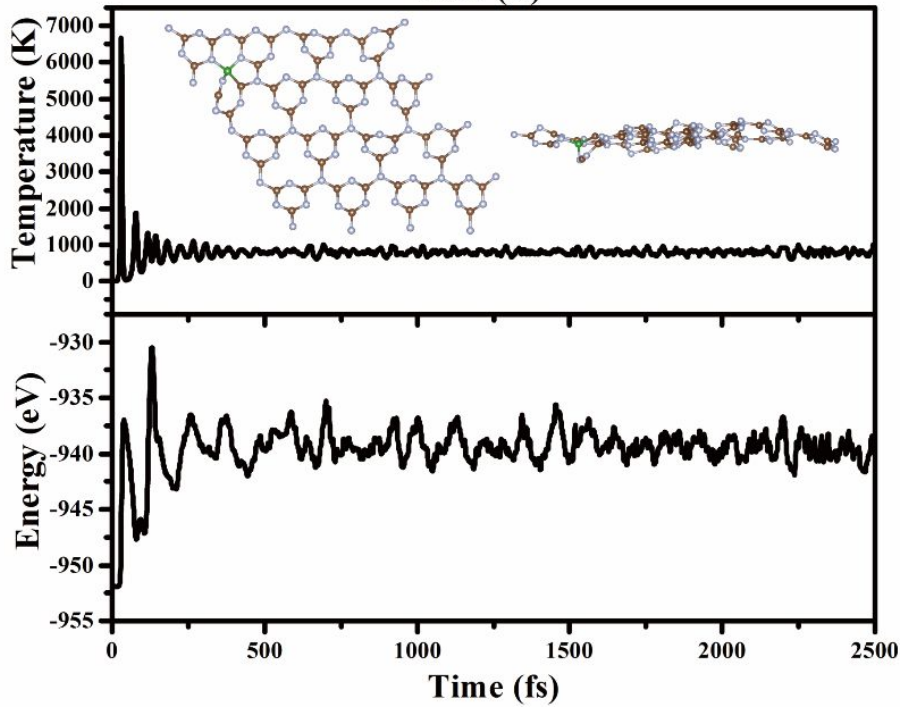

Figure S2. Changes of temperature and energy against the time for AIMD simulations of $\mathrm{B} / \mathrm{gt}-\mathrm{C}_{3} \mathrm{~N}_{4}$ modeled by a supercell of $(4 \times 4)$ run under different temperature: a) $400 \mathrm{~K}$, b) $600 \mathrm{~K}$ and c) $800 \mathrm{~K}$, inserts are top and side views of the snapshot of atomic configuration at $2.5 \mathrm{ps}$. 


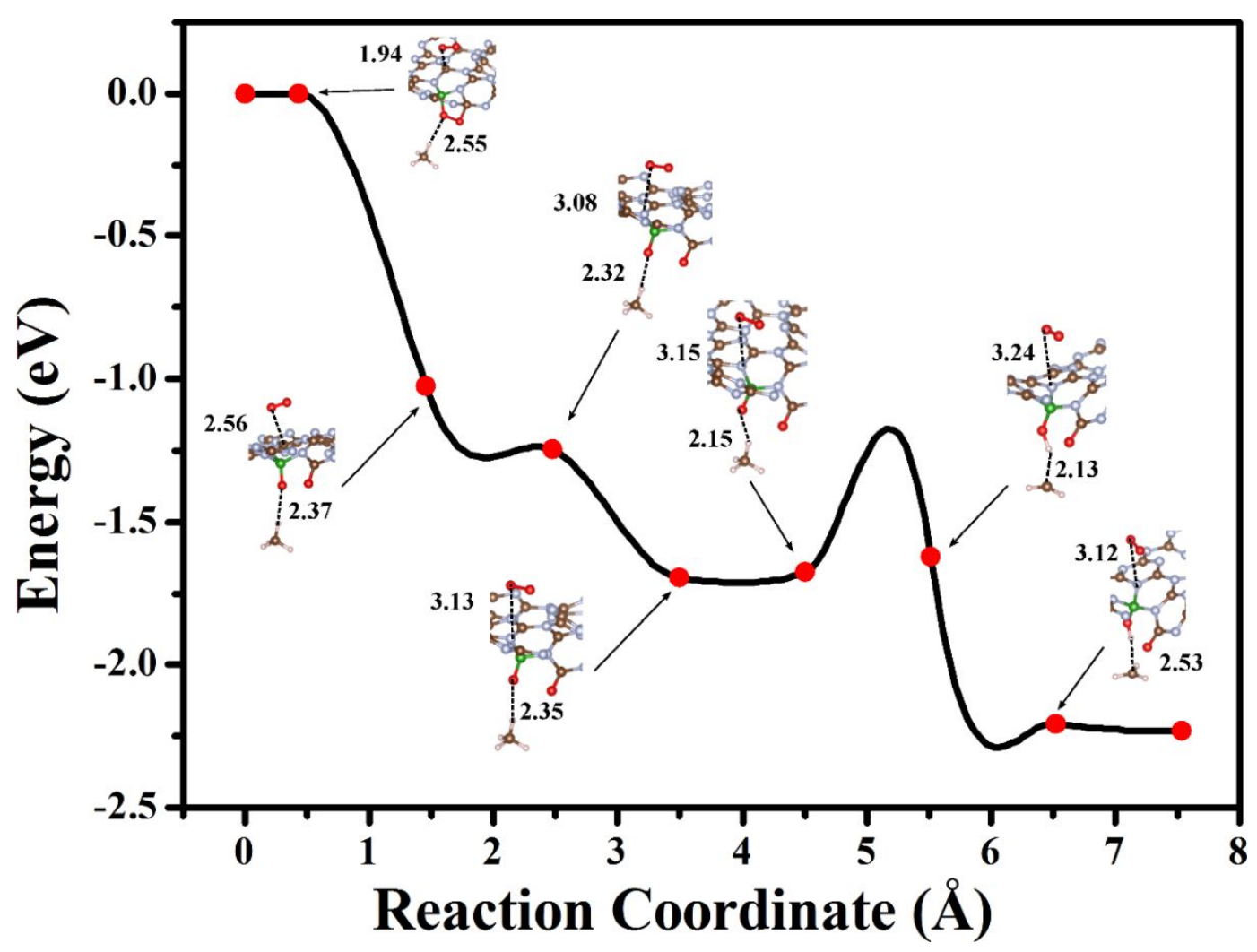

Figure S3. Calculated MEP for the H-abstraction from the adsorption of one methane molecule.
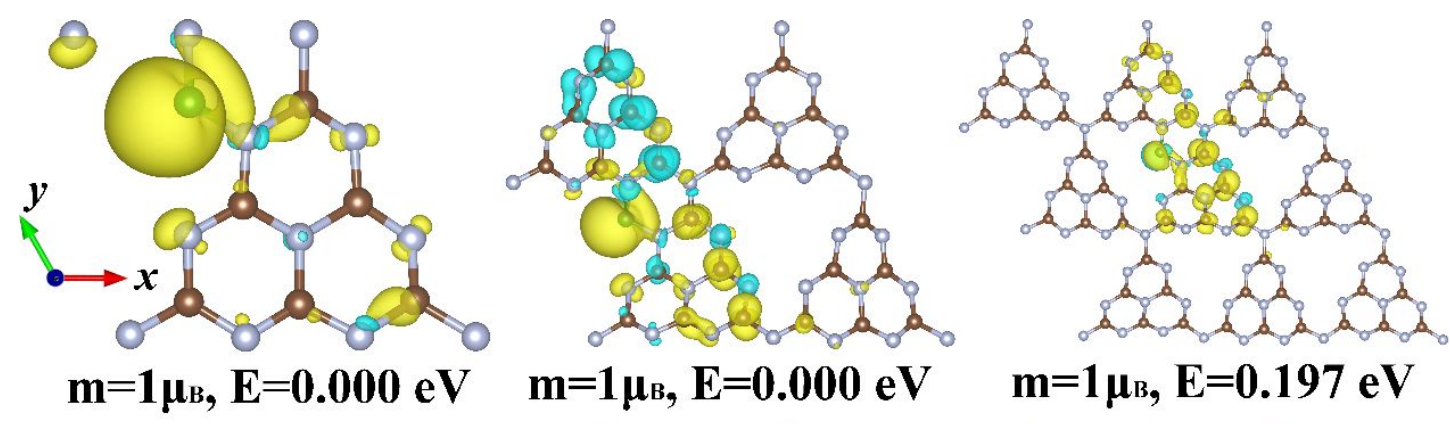

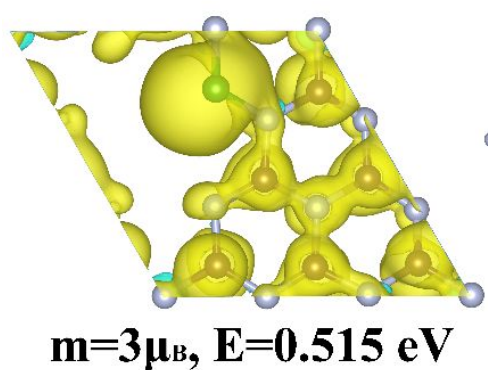

(a)

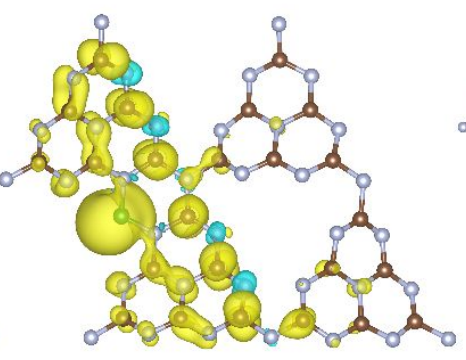

$\mathrm{m}=3 \mu_{\mathrm{B}}, \mathrm{E}=\mathbf{0 . 0 1 8 \mathrm { eV }}$

(b) $\mathrm{m}=1 \mu_{\mathrm{B}}, \mathrm{E}=0.197 \mathrm{eV}$

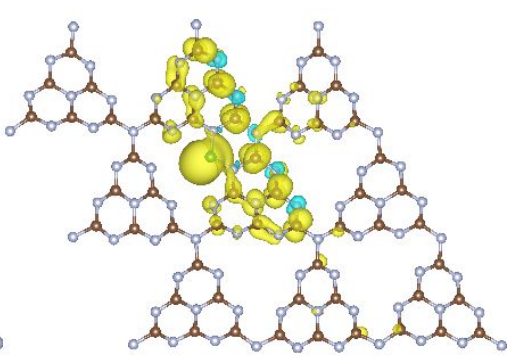

$\mathrm{m}=3 \mu_{\mathrm{B}}, \mathrm{E}=\mathbf{0 . 0 0 0} \mathrm{eV}$

(c)

Figure S4. Magnetic moment, relative energies and spin-polarized electron density of $\mathrm{B} / \mathrm{gh}-\mathrm{C}_{3} \mathrm{~N}_{4}$ modeled by different supercells: (a) $1 \times 1$, (b) $2 \times 2$ and (c) $3 \times 3$. The isosurface value is set to be 0.002 electron $/ \AA^{3}$ and the positive and negative densities are shown in yellow and cyan, respectively. The brown, gray and green balls represent the $\mathrm{C}, \mathrm{N}$ and $\mathrm{B}$ atoms, respectively. 

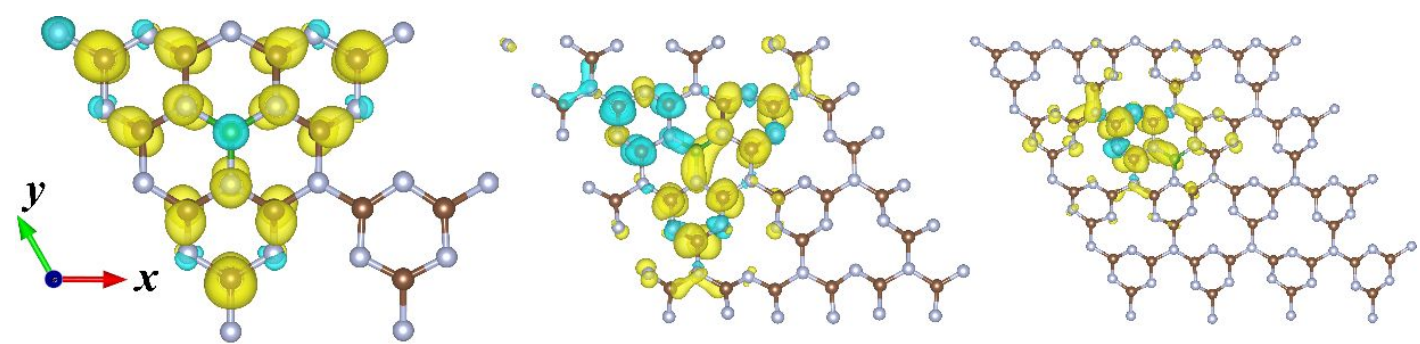

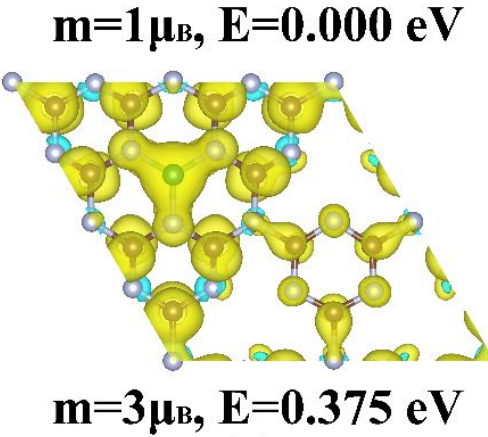

(a)

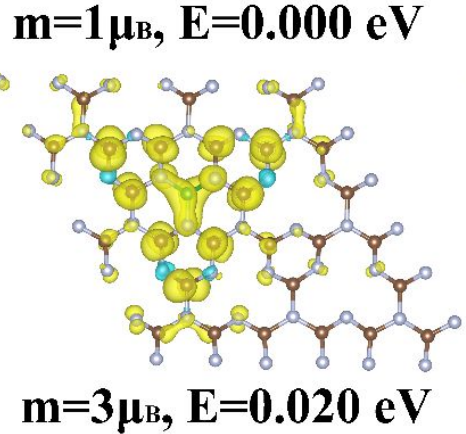

(b)

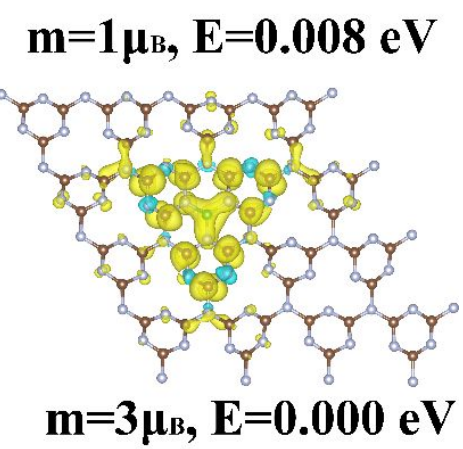

(c)

Figure S5. Magnetic moment, relative energies and spin-polarized electron density of $B / g t-C_{3} \mathrm{~N}_{4}$ modeled by different supercells: (a) $2 \times 2$, (b) $3 \times 3$ and (c) $4 \times 4$. The isosurface value is set to be 0.002 electron $/ \AA^{3}$ and the positive and negative densities are shown in yellow and cyan, respectively.

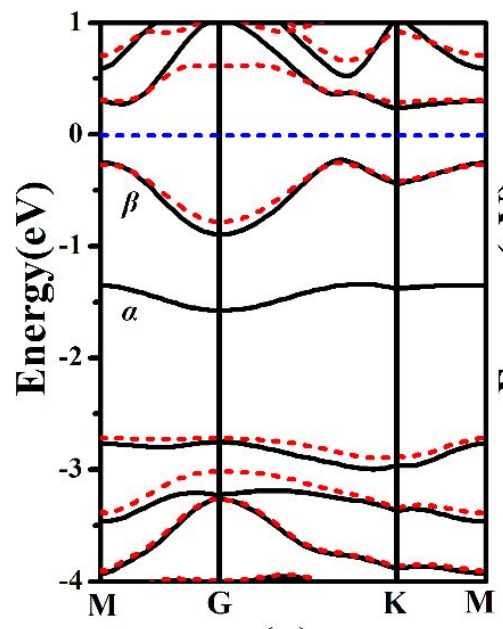

(a)

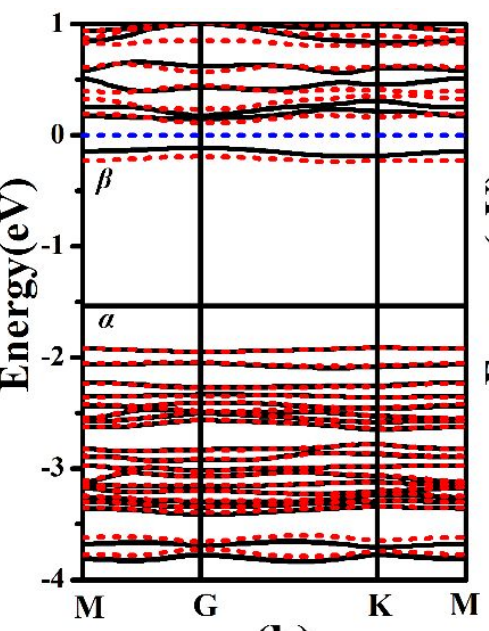

(b)

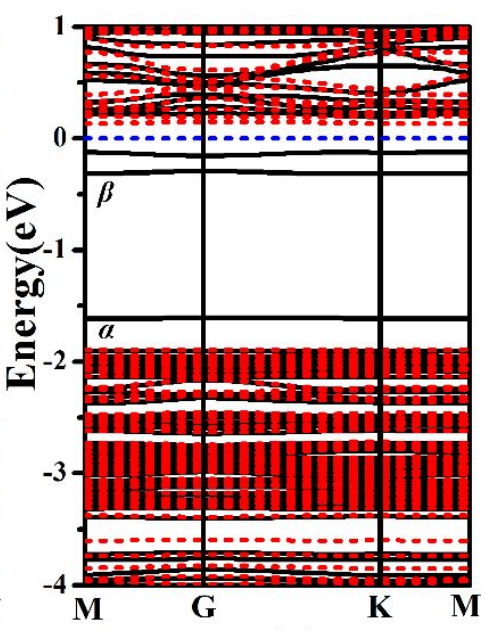

(c)

Figure S6. Spin-resolved band structure of $\mathrm{B} / \mathrm{gh}-\mathrm{C}_{3} \mathrm{~N}_{4}$ modeled by different supercells: (a) $1 \times 1$, (b) $2 \times 2$ and (c) $3 \times 3$. The black and red lines denote up- and down spin states, respectively. The blue and black dashed lines represent the Fermi energy.

When the single B atom doping in it, it realizes a P-type doping and introduces an isolated in-gap state (labelled as $\alpha$ in Fig. S6), locates below the Fermi level $\left(\mathrm{E}_{\mathrm{F}}\right)$ about $1.5 \mathrm{eV}$. Or to say, the Bdoping lets the $\mathrm{E}_{\mathrm{F}}$ of pristine gh- $\mathrm{C}_{3} \mathrm{~N}_{4}$ shift up about $1.5 \mathrm{eV}$ to move into the conduction band (CB) region, making the low-energy $\mathrm{CB}$ state (labelled as $\beta$ ) of the gh- $\mathrm{C}_{3} \mathrm{~N}_{4}$ present below the $\mathrm{E}_{\mathrm{F}}$ of $\mathrm{B} / \mathrm{gh}$ $\mathrm{C}_{3} \mathrm{~N}_{4}$. This means that significant changes happen only in this low-energy $\pi$ state of the pristine system. Although the $\alpha$ state in $1 \times 1 \mathrm{~B} / \mathrm{gh}-\mathrm{C}_{3} \mathrm{~N}_{4}$ shows some delocalized characteristics due to the 
interaction between adjacent $\mathrm{B}$ atoms, it still can induce the local magnetism of $1.0 \mu_{\mathrm{B}}$. With decreasing the doping concentration, the $\alpha$ state becomes a flat band in $2 \times 2$ system because of the weakened interaction between adjacent $B$ atoms. Meanwhile, the $\beta$ state becomes more localized, but it has not resulted in any enhancement on the magnetism due to that completely spin-splitting has not occurred yet. As the doping concentration decreases further, the $\beta$ state becomes totally localized in $3 \times 3$ system. Doubtlessly, spin degeneration of such localized states near the Fermi level is forbidden due to Coulomb repulsion (Ref 67 and 68), and thus spin-splitting occurs. More importantly, spin-flipping happens in the spin-split $\beta$ state, due to the altered spin-configuration from AFM to FM coupling, which enhances the magnetism by $2.0 \mu_{\mathrm{B}}$, directly resulting in the 3.0 $\mu_{\mathrm{B}}$ in the system.

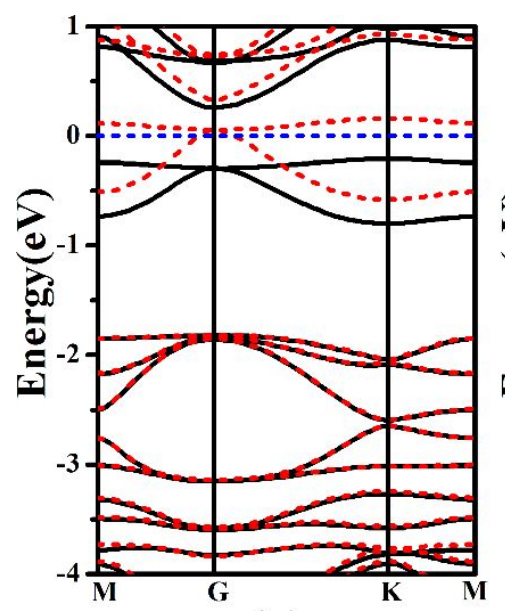

(a)

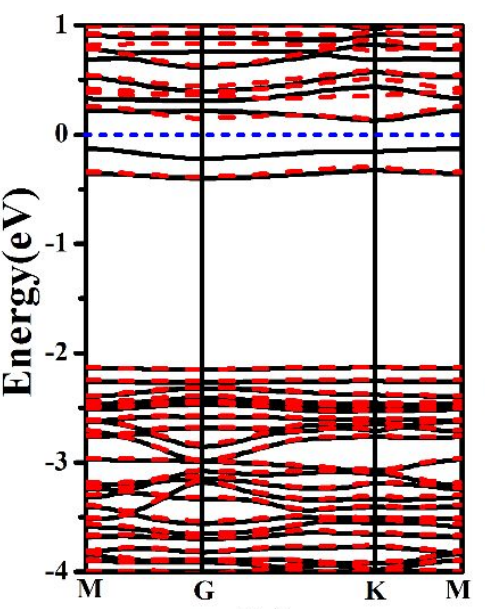

(b)

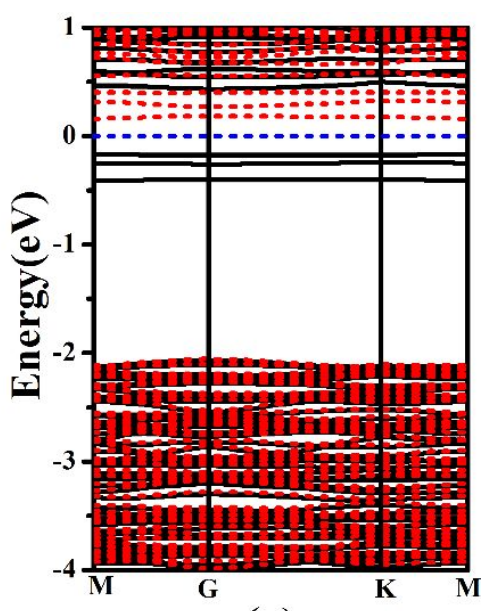

(c)

Figure S7. Spin-resolved band structure of B/gt- $\mathrm{C}_{3} \mathrm{~N}_{4}$ modeled by different supercells: (a) $2 \times 2$, (b) $3 \times 3$ and (c) $4 \times 4$. The black and red lines denote up- and down spin states, respectively. The blue and black dashed lines represent the Fermi energy.

As shown in this figure, B-doping in gt- $\mathrm{C}_{3} \mathrm{~N}_{4}$ cannot introduce an isolated in-gap state but induces an isolated doping state near the Fermi level (see band structures in Fig. S7), which gives rise to a magnetic moment of $1.0 \mu_{\mathrm{B}}$. Meanwhile, the B atom interrupted a $\pi$ bond of the pristine gt- $\mathrm{C}_{3} \mathrm{~N}_{4}$, giving rise to an additional magnetic moment of 0 or $2 \mu_{\mathrm{B}}$, depending on the configuration of netspin coupling. Hence, only the magnetism with spin moment of $1.0 \mu_{\mathrm{B}}$ or $3.0 \mu_{\mathrm{B}}$ presents in $\mathrm{B} / \mathrm{gt}-$ $\mathrm{C}_{3} \mathrm{~N}_{4}$ systems. 


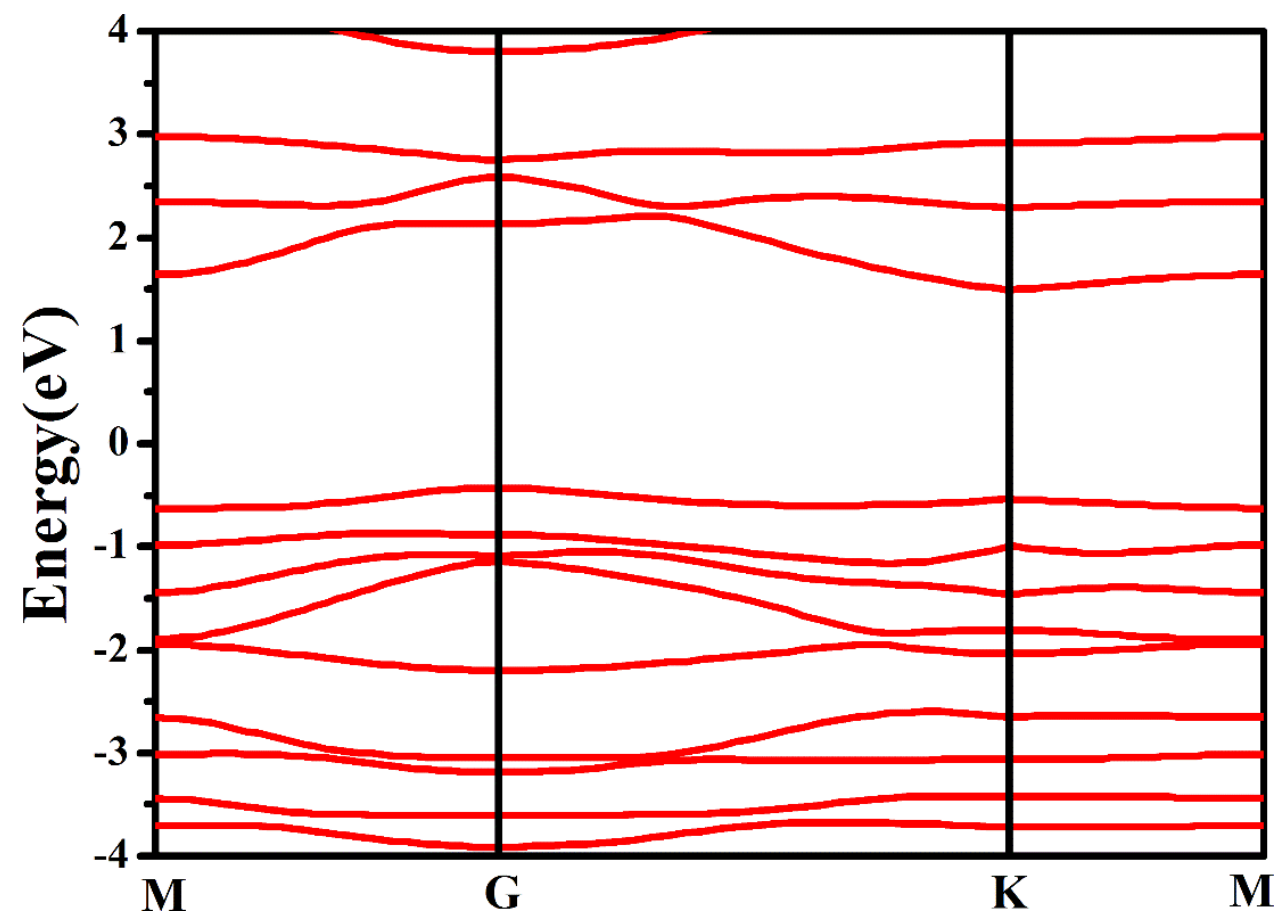

Figure S8. Band structures of bare corrugated gh- $\mathrm{C}_{3} \mathrm{~N}_{4}$. The Fermi level is set to zero. 